\section{Using nonlinear regression to estimate parameters of dark adaptation}

\author{
GERALD MCGWIN, JR., GREGORY R. JACKSON, \\ and CYNTHLA OWSLEY \\ University of Alabama, Birmingham, Alabama
}

An objective technique for estimating the kinetics of dark adaptation is presented, with which one can evaluate models with multiple parameters, evaluate several models of dark adaptation simultaneously, and rapidly analyze large data sets. Another advantage is the ability to simultaneously estimate transition times and rates of sensitivity recovery. Finally, this nonlinear regression technique does not require that the distributional properties of the data be transformed, and thus, parameter estimates are in meaningful units and reflect the actual rate of recovery of sensitivity.

In recent years, researchers have shown renewed interest in using dark adaptometry as a tool with which to investigate the mechanisms of aging (e.g., Coile \& Baker, 1992; Jackson, Owsley, \& McGwin, 1999), retinal disease (e.g., Brown, Adams, \& Coletta, 1986; Cideciyan, Pugh, Lamb, Huang, \& Jacobson, 1997; Holopigian, Seiple, Greenstein, Kim, \& Carr, 1997; Jacobson et al., 1995), and phototransduction (e.g., Cideciyan et al., 1998; Lamb, Pugh, Cideciyan, \& Jacobson, 1997; Leibrock, Reuter, \& Lamb, 1998). However, as we will discuss below, there are significant challenges in estimating the critical parameters of dark adaptation from data.

Dark adaptation is the recovery of light sensitivity by the retina in the dark following exposure of the photopigment to intense light (Barlow, 1972; Hecht, 1937). The typical dark adaptation paradigm consists of exposing the subject to a preadapting light, followed by measuring thresholds in the dark for $30-40 \mathrm{~min}$. The dark adaptation curve is produced by plotting log sensitivity as a function of time, as is illustrated in Figure 1, and is a biphasic function. Sensitivity is expressed in terms of $\log _{10}$ of sensitivity, since the relationship between the input to the receptor (i.e., light intensity) and the receptor output is a logarithmic one (see Cornsweet, 1970). The first and faster phase of dark adaptation represents the cone-mediated contribution. The slower and longer second phase of the dark adaptation function is the rod-mediated contribution. The inflection point between the two phases is known as the rod-cone break, which represents the point in time during dark adap-

This research was funded by National Institutes of Health Grants R01 AG04212 and T32 EY07033, by a department grant from Research to Prevent Blindness, Inc., and by the Alabama Eye Institute. Correspondence concerning this article should be addressed to $\mathrm{C}$. Owsley, Department of Ophthalmology, School of Medicine, University of Alabama, 700 S. 18th St., Birmingham, AL 35294-0009 (e-mail: owsley@. eyes.uab.edu). tation at which the recovering rods' sensitivity surpasses the cones' sensitivity.

Recent advances in our understanding of the mechanisms underlying dark adaptation have identified additional biologically relevant parameters of dark adaptation, such as the time constants of the second and third components of rod-mediated dark adaptation (Leibrock et al., 1998). The rates of recovery of sensitivity during the second and third component of dark adaptation are largely dictated by the rate of rhodopsin regeneration, as has been indicated by electrophysiological work on animal models (Baylor, Matthews, \& Yau, 1980; Dowling, 1960; Lamb, $1980,1981)$ and retinal densitometry findings in humans (Rushton, Campbell, Hagins, \& Brindley, 1955). On the basis of these findings, a biologically plausible model of dark adaptation can be constructed to describe the sensitivity recovery of the visual system during dark adaptation. This model consists of a single exponential representing the cone-mediated sensitivity recovery and two linear components representing the second and third components of rod-mediated sensitivity recovery. The rodcone break and the transition point in time between the second and the third components must also be estimated.

Quantifying parameters of dark adaptation on the basis of these biologically based, multicomponent models can be difficult. This is primarily attributable to the fact that it is necessary to solve for several parameters simultaneously, each of which is dependent on the other parameters to be solved. Furthermore, the statistical models used to solve for these parameters are not mathematically simple (as in a single-exponential decay; see Hahn \& Geisler, 1995), because, in the present situation, we are simultaneously fitting the time constants of three components and two transition points. Furthermore, this analytic problem cannot be easily solved with popular off-the-shelf, curvefitting software. For these reasons, it is not surprising that the literature on dark adaptation is devoid of studies using sophisticated analytic techniques for identifying component parameters. We developed a nonlinear regression modeling technique for estimating parameters of dark adaptation that overcomes these problems and can be easily implemented. Not only does this technique minimize experimenter bias, but it facilitates the analysis of large sample studies, such as those addressing development or aging trends or those used in clinical trials on retinal dysfunction. Especially in the case of clinical trials, one needs a standard and objective methodology that can be used with a wide range of patients with varying disease severity.

\section{Application of Nonlinear Regression to Dark Adaptation Data}

The goal of nonlinear regression is to fit a model to a set of data (Bard, 1974; Gallant, 1975; McCullagh \& Nelder, 1983). This is a more general approach than those of other types of regression models (e.g., linear regres- 


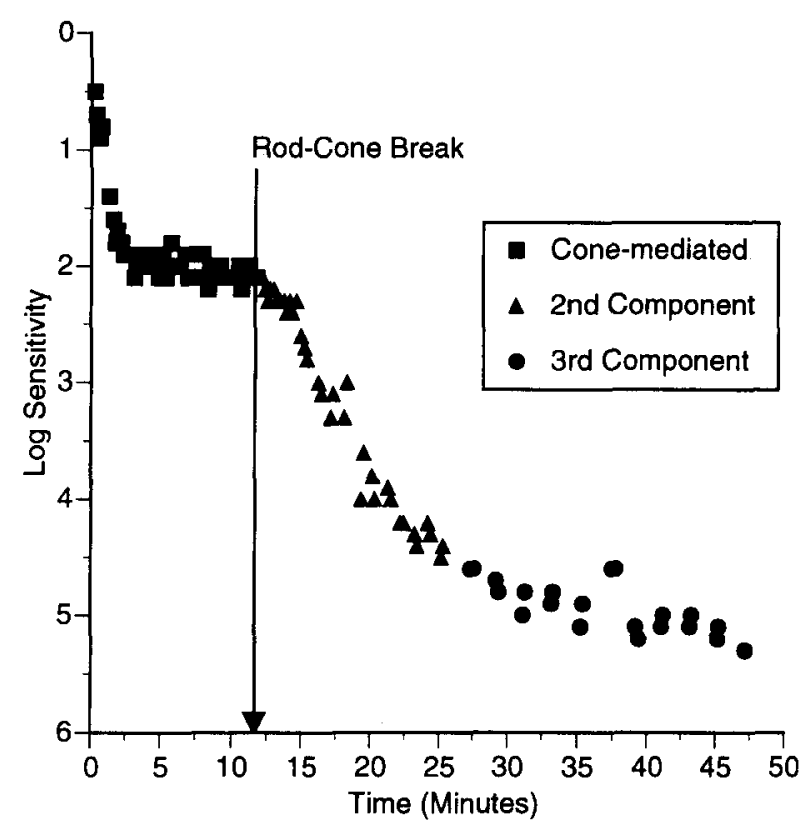

Figure 1. Typical dark adaptation curve illustrating different underlying components of the dark adaptation process.

sion), wherein one is applying a specific, preconceived model to a set of data. Nonlinear regression can fit data to any equation that defines $Y$ as a function of $X$ and one or more variables. It finds the values of those variables that generate the curve that comes closest to the data. More precisely, the goal is to minimize the sum of the squares of the vertical distances of the points from the curve.

The application of nonlinear regression to dark adaptation data offers several features that are well suited for analyzing this type of data. Nonlinear regression allows for the use of data expressed in terms of its actual distribution. This can be done because it is possible to specify the exact form of an equation, rather than attempting to manipulate the data (e.g., changing the data's distributional properties through transformation) to fit the form of an equation. For example, it is not uncommon for researchers to mathematically transform data to make it fit a Gaussian distribution, so that linear models can be applied to it. A more appropriate approach would be to use the actual, untransformed data and develop a regression model that reflects the true structure of the data. Therefore, parameter estimates are in meaningful units and do not require back transformations for interpretation purposes. Another feature of nonlinear regression is the ability to simultaneously estimate transition times and rates of recovery free of experimenter bias. It can accomplish this by treating such parameters as variables in the model and iteratively solving for them until a specified stopping point (usually, until adjustments make virtually no difference in the sum of squares). Finally, any model of dark adaptation based on a theoretical or a biological construct that can be expressed mathematically can be estimated with this technique.

To implement this nonlinear regression technique, we used SAS (SAS Institute, Cary, NC). Although a number of other software packages (e.g., Matlab [The Mathworks, Inc., Natick, MA], S-Plus [MathSoft, Inc., Cambridge, MA]) could have been used, SAS was chosen for the following reasons. First, SAS is a popular and widely used software package in the behavioral sciences. Second, using built-in statistical procedures, we were able to easily implement the nonlinear regression portion of the program. Third, SAS is a cross-platform software package (e.g., PC, Macintosh, Unix), making this program easily implemented in numerous computing environments without modification. Fourth, SAS offers a flexible framework that allows the evaluation of multiple models without extensive modification to the analysis program.

\section{Description of the Program}

The data input (see the program listing in the Appendix) portion of the program reads a tab-delimited text file specified by the infile command. The order of the variables in the file is subject identification number (ID), time (MIN), and log threshold (THRESH). The program initialization portion of the program creates temporary variables and conducts preliminary calculations to be used later in the program.

The model initialization portion of the program conducts a preliminary linear regression analysis. In SAS, the nonlinear regression procedure requires that the user supply the initial values for parameter estimates. This is accomplished by using the results of a preliminary linear regression. The preliminary linear regression models the dark adaptation data, using four linear components. The user chooses the four linear components as delimited by the inputted transition points. These points are fixed and are not explicitly solved as part of the analysis. An alternative approach would have been to exclude the preliminary linear regression modeling and fix the initial values for the nonlinear regression procedure. However, the drawback of this alternative is apparent when there is large individual variability in the data (e.g., normal vs. those with retinal disease). By running the linear regressions individually for each subject, the resulting nonlinear regressions are provided initial values that are specific to that subject, thereby reducing computing time by decreasing the number of computational iterations necessary to solve for the final nonlinear model parameters.

The nonlinear regression portion of the program contains four statements for each model evaluated. The resulting values from the linear regression model are used to initialize the parameter estimates in the PARMS statement of the PROC NLIN procedure. The preliminary transition times (KNOTS) are specified by the user. It should be noted that these KNOT values are solved as part of the model estimation procedure and are not treated as fixed values. For estimation of the model parameters, the 


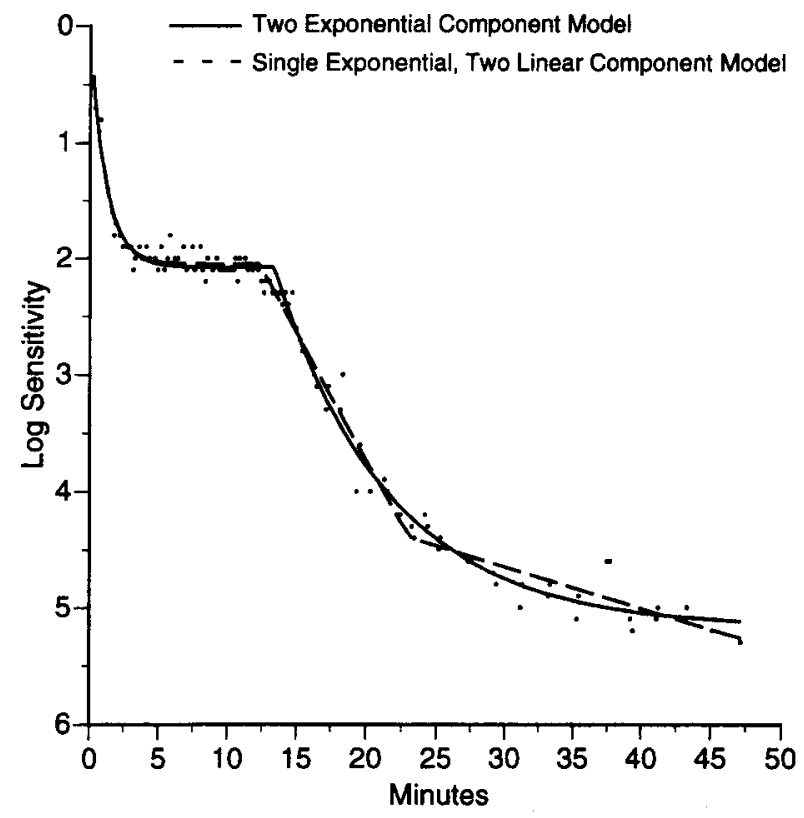

Figure 2. Dark adaptation curve from a 25-year-old person in good ocular health. Solid line represents the nonlinear regression fits for the two-exponential model, and dashed line represents the oneexponential, two-linear model.

NLIN procedure uses the method of false position (also known as the doesn't use derivatives [DUD] or secant method; Ralston \& Jennrich, 1978). One could also use a number of other estimation procedures, including GaussNewton, Marquardt, steepest descent (gradient), and Newton (Bard, 1974; Hartley, 1961; Marquardt, 1963). To use the Gauss-Newton method, delete the METHOD= DUD option in the PROC NLIN statement. Our evaluation of these methods indicated that they produced equivalent results, with the method of false position requiring slightly fewer iterations. The MODEL statement is the mathematical expression of the dark adaptation model. The NLIN procedure iterates a maximum of 200 times. Estimation of model parameters will cease when the change in the sum of squared errors from iteration ${ }^{j-1}$ to iteration $^{j}$ is $10^{-8}$ or less. The $r^{2}$ value is also calculated for each model. Finally, the subject number, solved parameters, and $r^{2}$ value are output to the screen. In the case of multiple subjects, the program then processes the next subject's data.

\section{Examples}

We demonstrate the application of nonlinear regression on data from two observers, a 25 -year-old and a 75 year-old, both in good ocular health. Two models of dark adaptation are applied to each subject's data: a classic two-exponential-component model $[y=(a+b * \exp (-c$ $* x))+(d * \exp (-e *([\max (x-\operatorname{knot} 1,0)])))$; Barlow, 1972; Hecht, 1937] and a single-exponential, two-linearcomponent model of dark adaptation $[y=((a+b *$ $\exp (-c * x))+(d *([\max (x-\operatorname{knot} 1,0)])))+(e *$ ([max $(x-\operatorname{knot} 2,0)]))$; based on Lamb, Cideciyan, Jacobson, \& Pugh, 1998, and Leibrock et al., 1998].

Figure 2 presents $\log$ sensitivity as a function of time after the bleach for the 25-year-old adult. This data is representative of adults in their 20s (Jackson et al., 1999). The nonlinear regression program was applied to the data; the solved nonlinear regression equations for both the two-exponential-component model (solid line) and the one-exponential, two-linear-component model (dashed line) are plotted. The equation for the former model is $y=51.71+(-19.78 * \exp (-0.85 * x))+$ $(-30.94 * \exp (-0.12 *(\operatorname{MAX}(x-13.32,0))))$, with an $r^{2}$ value of .99 . For the latter model, the equation is $y=$ $20.49+(-19.86 * \exp (-0.91 * x))+(2.17 *(\operatorname{MAX}(x-$ $12.23,0)))+(-1.81 *(\operatorname{MAX}(x-23.06,0)))$. This model had an $r^{2}$ value of .99 . Both models fit the data well, as is evidenced by the high $r^{2}$ values. A $z$ test can be used to determine whether one model provides a significantly better fit to the data. There was no evidence that either model fit the data better $(z=0.06, p>.05)$.

Dark adaptation data and solved nonlinear regression equations for the 75-year-old subject are presented in Figure 3. Respectively, the equations for the two-exponentialcomponent model and the one-exponential, two-linearcomponent model are $y=47.87+(-15.68 * \exp (-0.2 *$ $x))+(-29.78 * \exp (-0.09 *(\operatorname{MAX}(x-18.32,0))))$ and $y=14.65+(-398.54 * \exp (-1.77 * x))+(1.54 *(\operatorname{MAX}(x$ $-14.21,0)))+(-1.28 *(\operatorname{MAX}(x-31.4,0)))$. As is evident from Figure 2, the two-exponential-component model does not appear to fit the data as well as the one-exponential, twolinear-component model. This is supported by the higher $r^{2}$

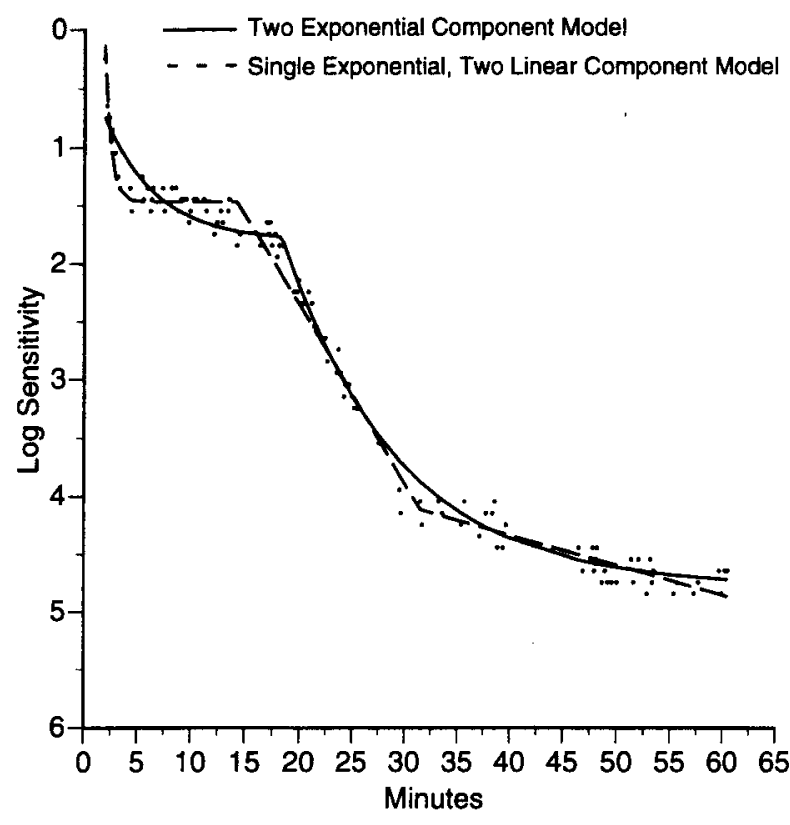

Figure 3. Dark adaptation curve from a 75-year-old person in good ocular health. Solid line represents the nonlinear regression fits for the two-exponential model, and dashed line represents the oneexponential, two-linear model. 
value for the one-exponential, two-linear-component model (.99), as compared with that of the two-exponential-component model (.92). The one-exponential, two-linear-component model fits the data better than does the two-exponential-component model $(z=5.77, p<.001)$.

The values for the transition times are available directly from the program output. For example, for the twoexponential-component model for the 75-year-old subject, the transition time was $18.32 \mathrm{~min}$. To obtain rates of sensitivity recovery, simple mathematical calculations are performed on the parameter estimates. The nature of these calculations are dependent on the form of the dark adaptation model chosen.

\section{Availability}

Interested users who prefer not to type in the programs can request an ASCII file of the program by e-mailing the first author (mcgwin@eyes.uab.edu).

\section{REFERENCES}

BARD, J. (1974). Nonlinear parameter estimation. New York: Academic Press.

Barlow, H. B. (1972). Dark and light adaptation: Psychophysics. In D. Jameson \& L. M. Hurvich (Eds.), Handbook of sensory physiology (Vol. VII, pp. 1-28). New York: Springer-Verlag.

Baylor, D. A., Matthews, G., \& Yau, K. W. (1980). Two components of electrical dark noise in toad retinal rod outer segments. Journal of Physiologv, 309, 591-621.

Brown, B., Adams, A. J., \& ColetTA, N. J. ( 1986). Dark adaptation in age-related maculopathy. Ophthalmologic \& Physiologic Optics, 6 , $81-84$.

Cideciyan, A. V., Pugh, E. N., Lamb. T. D., Huang, Y.. \& Jacobson. S. G. ( 1997). Rod plateau during dark adaptation in Sorsby's fundus dystrophy and vitamin A deficiency. Investigative Ophthalmology \& Visual Science, 38, 1786-1794.

Cideciyan, A. V., Zhao, X. Y., Nifisen, L., Khani, S. C., Jacobson, S. G., \& PAlczewski, K. (1998), Null mutation in the rhodopsin kinase gene slows recovery kinetics of rod and cone phototransduction in man. Proceedings of the National Academy of Sciences, 95, 328-333.

Colle, C. D., \& BAker, H. D. (1992). Foveal dark adaptation, photopigment regeneration, and aging. Visual Neuroscience, 8, 27-29.
Cornsweet, T. N. (1970). Visual perception. New York: Academic Press.

DowLing, J. E. (1960). The chemistry of visual adaptation in the rat. Nature, 188, 114-118.

Gallant, A. R. (1975). Nonlinear regression. American Statistician, 29, 73-81

HAHN, L. W., \& GeISLER, W. S. (1995). Adaptation mechanisms in spatial vision: I. Bleaches and backgrounds. Vision Research, 35, 1585 1594.

Hartley, H. O. (1961). The modified Gauss-Newton method for the fitting of nonlinear regression functions by least squares. Technometrics, 3, 269-280.

HeCht, S. (1937). Rods, cones, and the chemical basis of vision. Physiological Review, 17, 239-290.

Holopigian, K., Seipie, W., Greenstein, V., Kim, D., \& Carr, R. E. (1997). Relative effects of aging and age-related macular degeneration on peripheral visual function. Optometry \& Vision Science, 74, 152-159

Jackson, G. R., Owsley, C., \& McGwin, G. (1999). Aging and dark adaptation. Vision Research, 39, 3975-3982.

Jacobson, S. G., Cideciyan, A. V., Regunath. G., Rodriguez, F. J., Vandenburgh, K., Sheffield, V. C., \& Stone, E. M. (1995). Night blindness in Sorsby's fundus dystrophy reversed by vitamin A. $\mathrm{Na}$ ture Genetics, 11, 27-32.

LAMB, T. D. (1980). Spontaneous quantal events induced in toad rods by pigment bleaching. Nature, 287, 349-351.

LAMB, T. D. (1981). The involvement of rod photoreceptors in dark adaptation. Vision Research, 21, 1773-1782.

Lamb, T. D., Cideciyan, A. V., Jacobson, S. G., \& Pugh, E. N. (1998). Towards a molecular description of human dark adaptation. Journal of Physiology, 506, 88P.

Lamb, T. D., Pugh, E. N., Cideciyan, A. V., \& Jacobson, S. G. (1997). A conceptual framework for analysis of dark adaptation kinetics in normal subjects and in patients with retinal disease. Investigative Ophthalmology \& Vision Science, 38(Suppl.), S1121.

Leibrock, C. S., Reuter, T., \& LAMB, T. D. (1998). Molecular basis of dark adaptation in rod photoreceptors. Eye, 12, 511-520.

MARQUARDT, D. W. (1963). An algorithm for least squares estimation of nonlinear parameters. Journal for the Society of Industrial \& Applied Mathematics, 11, 431-441.

McCullagh, P., \& Nelder, J. A. (1983). Generalized linear models. London: Chapman Hall.

RALSTON, M. L., \& JENNRICH, R. I. (1978), DUD, a derivative-free algorithm for nonlinear least squares. Technometrics, 20, 70-74.

Rushton, W. A. H., Campbell, F. W., Hagins, W. A., \& Brindley, G. S. (1955). The bleaching and regeneration of rhodopsin in the living eye of the albino rabbit and of man. Optical Acta, 1, 183-190. 


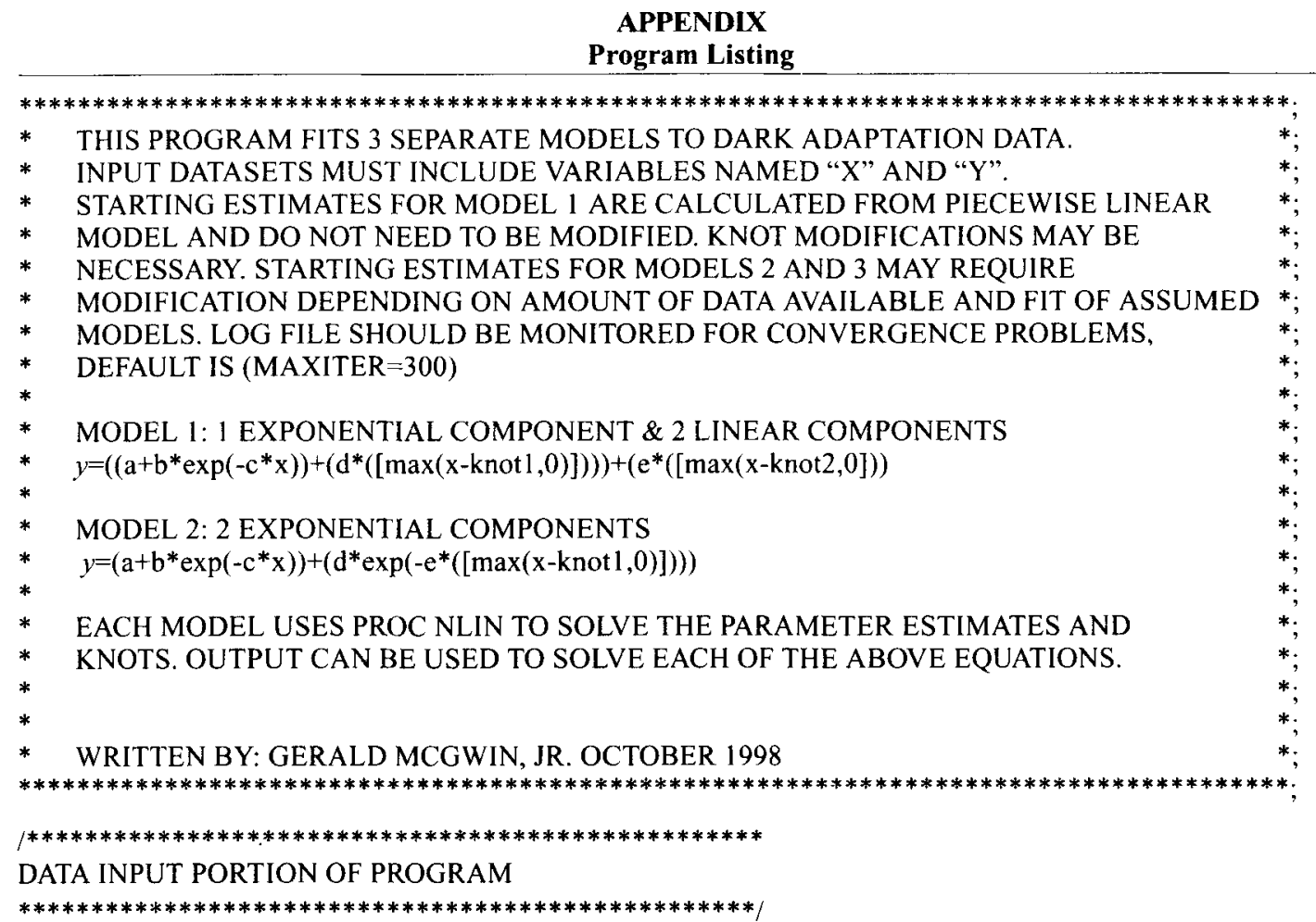

data one;

infile "c: $\backslash$ testdata.txt" expandtabs missover;

input ID MIN THRESH;

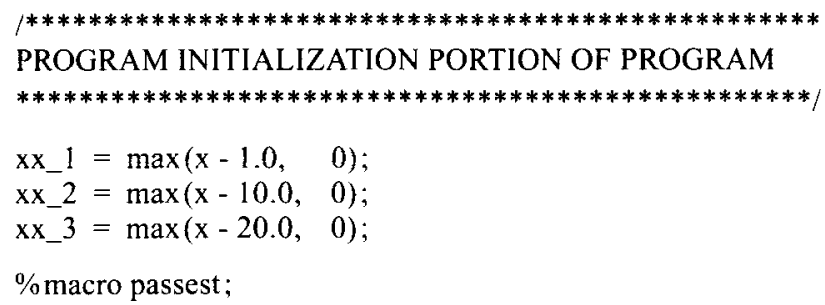

proc univariate noprint; var $y$; output out $=$ tss_temp mean $=$ ave ;

data_null_; set tss_temp;

call symput('ave',put(ave,12.6));

data aa; set one;

temp $=(\mathrm{y}-\& \text { ave })^{* *} 2$

proc univariate noprint; var temp; output out=tss sum $=t s s$;

data_null_; set tss;

call symput('tss',put(tss,8.6));

proc reg noprint data $=$ one outest $=$ reg_est; model $y=x x x \_1$ xx_2 $x x \_3$;

data_null_; set reg_est;

call symput('b0',put(intercep,5.2));

call symput('b1',put $(x, 5.2))$;

call symput('b2',put( $\left.\left.x x_{-} 1,5.2\right)\right)$;

call symput('b3',put( $\left.\left.x x \_2,5.2\right)\right)$;

call symput(' $b 4$ ',put( $\left.x x \_3,5.2\right)$ ); 


\section{APPENDIX (Continued)}

$* * * * * * * * * *$.

proc $n$ lin noprint $\operatorname{maxiter}=300$ method $=$ dud data $=$ one outest $=$ nlin_est ;

parms $a=20 b=-20 \mathrm{c}=0.90 \mathrm{~d}=2.10 \mathrm{e}=0.40 \mathrm{knot} \mathrm{l}=10 \mathrm{knot} 2=23$;

$\mathrm{xx} 1=\max (\mathrm{x}-\mathrm{knot} 1,0) ;$

$\mathrm{xx} 2=\max (\mathrm{x}-\mathrm{knot} 2,0)$;

model $y=\left(\left(a+b^{*} \exp \left(-c^{*} x\right)\right)+\left(d^{*}(x \times 1)\right)\right)+\left(e^{*}(x \times 2)\right) ;$

data nlin_est; set nlin_est; if_type__= "FINAL"; drop_name__iter_; model $=$ "1 EXP \& 2 LINE" ; r2=1-(_sse_/\&tss);

proc print data $=$ nlin_est;

$* * * * * * * * * *$;

proc nlin noprint maxiter $=300$ method $=$ dud data $=$ one outest $=$ nlin_est;

parms $a=50 b=-20 \mathrm{c}=0.8 \mathrm{~d}=10 \mathrm{e}=0.2 \operatorname{knot} \mathrm{l}=15$;

$\mathrm{xx}=\max (\mathrm{x}-\mathrm{knot} \mathrm{l}, 0)$;

model $y=\left(a+b^{*} \exp \left(-c^{*} x\right)\right)+\left(d^{*} \exp \left(-e^{*}(x x)\right)\right)$

data nlin_est; set nlin_est; if_type_ = "FINAL"; drop_name__iter_; model $=$ "2 EXPONENTIALS"; r2=1-(_sse_/\&tss);

proc print data $=$ nlin_est ;

$* * * * * * * * * *$;

$\%$ mend passest;

\%passest

run; 